\title{
Chest pain, enzymes and hypothyroidism
}

\author{
S R Strachan, O Afolabi, N Brown, D Gray
}

\begin{abstract}
Summary
Hypothyroidism is a common disorder and when presenting with classical symptoms and signs is easy to recognise. However, hypothyroidism may present in a manner suggestive of an acute myocardial infarction with an elevated creatine kinase and electrocardiographic abnormalities. We report a case of severe hypothyroidism presenting as a cardiac event whose symptoms and signs dispersed following treatment with thyroxine.
\end{abstract}

Keywords: hypothyroidism; chest pain; creatine kinase

Cardiology

Department, Queen's

Medical Centre,

University Hospital,

Clifton Boulevard,

Nottingham,

NG7 2UH, UK

S R Strachan

O Afolabi

N Brown

D Gray

Correspondence to S R Strachan, Intensive Care Unit, Queens Medical

Centre, Nottingham NG7

2UH, UK

Submitted 29 April 1999 Accepted 19 August 1999
A 55-year-old man attended casualty immediately on return from his holiday. He gave a 4 -week history of general malaise with pressure and aches in his chest, breathlessness on exertion and numbness in his little fingers. $\mathrm{He}$ denied nausea, vomiting, sweats, fevers, rashes or sore throat. He had full recovery from a possible stroke 5 years earlier with a normal computed tomography head scan. He was on no medication, drank moderately and had gained 7 pounds in weight in the past 6 months after

On examination he was tanned and of medium build. He appeared in some discomfort but was apyrexial, heart rate 60 beats/min, stopping smoking.

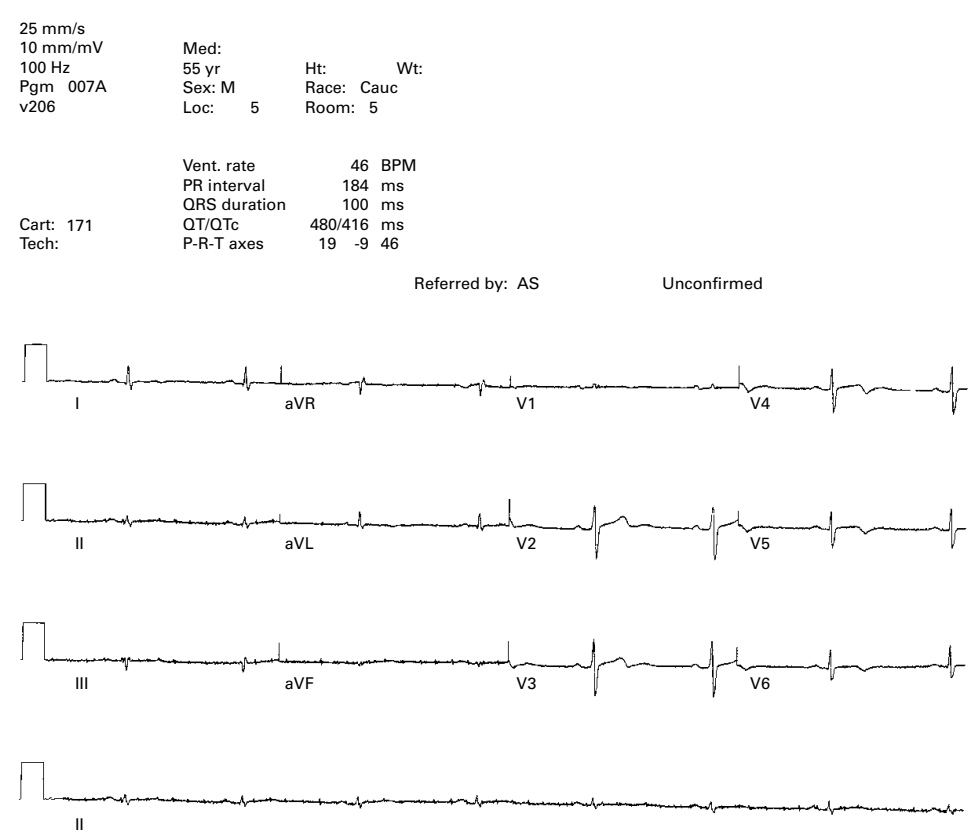

Figure ECG $6 \mathrm{~h}$ after admission

blood pressure $155 / 85 \mathrm{mmHg}$. There were no positive findings on examination. Initial investigations revealed a normal full blood count and a mildly raised urea and creatinine, markedly elevated creatine kinase (CK) and lactate dehydrogenase (LDH) at 1406 and $1080 \mathrm{IU} / 1$, respectively. Chest radiography was normal. He was in sinus rhythm with sequential ST/T wave change in the lateral chest leads on electrocardiography (ECG) 6 hours later (figure).

At this stage the differential diagnosis was of unstable angina, myocardial infarction or myocarditis; the patient was monitored overnight. Next day, CK had increased to $5750 \mathrm{IU} / 1$ and LDH to 1222 IU/1. Erythrocyte sedimentation rate and C-reactive protein were normal, as were alkaline phosphatase, bilirubin, and albumin, but alanine aminotransferase, aspartate aminotransferase and gamma-glutamyl transferase were mildly increased. Cholesterol level was $7.1 \mathrm{mmol} / \mathrm{l}$. Two days later his chest pain had improved although he still felt generally unwell. Cardiac troponin $\mathrm{T}$ was undetectable on bedside testing and echocardiography was essentially normal. In view of the myalgia, bradycardia and elevated CK, thyroid function tests were revealed a thyroid-stimulating hormone (TSH) of $82 \mathrm{nmol} / \mathrm{l}$ and undetectable free thyroxine levels. Thyroid auto-antibodies were strongly positive.

On further questioning the patient's wife described him as 'slowing down' for over a year and had attributed this to 'overwork'. In addition, it became clear that other members of his family had been treated for hypothyroidism; he had not mentioned this as their symptoms were quite different and no-one had asked him about his family before!

Thyroxine was commenced at a low initial dose $(25 \mu \mathrm{g})$ which rapidly led to marked improvement of symptomatology and normalisation of biochemistry. It was felt that as his symptoms entirely resolved with thyroid hormone replacement he did not have coronary artery disease, so further invasive cardiological investigations (eg, angiography) were not indicated.

\section{Discussion}

Typical features of hypothyroidism include lethargy, dry skin, hoarseness, constipation, cold intolerance, myxoedematous facies, weight gain and bradycardia. However, patients may present with atypical symptoms suggestive of myocardial infarction or a multisystem disorder such as polymyositis. In these instances investigations 
may be misleading so a high index of suspicion is required to consider hypothyroidism as the primary diagnosis.

It is well documented that hypothyroidism can cause persistent elevation of serum enzymes, the cause of which may be leakage from increased cell permeability. ${ }^{1} \mathrm{Up}$ to $90 \%$ of patients with hypothyroidism have a raised $\mathrm{CK}$ that resolves with thyroid hormone replacement ${ }^{2}$; on isoanalysis this is most commonly due to the CK-MM fraction, though CK-MB fraction may be elevated due to decreased renal clearance of $\mathrm{CK}$.

ECG changes including T-wave flattening or inversion, small $\mathrm{P}$ waves or $\mathrm{QRS}$ complexes and QRS widening have been reported in hypothyroidism. ${ }^{4}$ Hypercholesterolaemia predisposes to coronary heart disease and ischaemic heart disease and hypothyroidism may coexist. Troponin I is elevated within 6 hours of myocardial damage but is normal in hypo-

1 Doran GR, Wilkinson JH. The origin of the elevated activities of creatine kinase and other enzymes in the sera of patients with myxoedema. Clin Chim Acta 1975;62:203-11.

2 Lemar HJ, West SG, Garrett CR, Hofeldt FD Covert hypothyroidism presenting as a cardiovascular event. Am $\mathcal{F}$ Med 1991;91:549-52.

\section{Summary points}

- a diagnosis of hypothyroidism is not always easy

- hypothyroidism is a cause of elevated liver, muscle and cardiac enzymes - especially creatine kinase

- hypothyroidism can cause electrocardiographic abnormalities

- a thorough, detailed family history is important

thyroidism and consequently may distinguish true myocardial damage. ${ }^{3}$

Clinically, the diagnosis of hypothyroidism can be difficult. In patients presenting with features not entirely typical of ischaemic heart disease but with raised CK and/or ECG abnormalities a diagnosis of hypothyroidism needs to be considered. Myocardial infarction can be excluded confidently by troponin I testing. ${ }^{3}$

\footnotetext{
3 Cohen LF, Mohabeer AJ, Keffer JH, Jialal I. Troponin I in hypothyroidism. Clin Chem 1996;42:1494-5.

4 Hamolsky MW, Kurland GS, Freedberg AS. The heart in hypothyroidism. F Chron Dis 1961;14:558-69.
}

\footnotetext{
Answers to questions on $\mathbf{p} 132$

1 False. A reference range is derived from the mean and two standard deviations above and below it.

2 False. Plasma osmolality is normal in pseudohyponatraemia due to the presence of lipid or protein as the sodium concentration within the plasma water is normal. When the sample has been diluted by intravenous infusion fluid there is less sodium within the plasma water and the plasma osmolality will be approximately equal to the osmolarity, which can be calculated using the formula $2([\mathrm{Na}]+[\mathrm{K}])+[\mathrm{urea}]+[$ glucose $]$, and will therefore be low.

3 True. Due to the wide reference range for plasma creatinine and its inverse relationship with glomerular filtration rate.

4 True. Plasma glucose is greater than whole blood glucose by about $12 \%$.

5 True. Aspartate transaminase activity may be increased in hypothyroidism, as may CK activity.
} 